PEIXOTO JUNIOR, K.C., FIRMIANO, A.C. e CRESPILHO, A.M. Relação entre reprodução e produção de bovinos de leite. PUBVET, Londrina, V. 7, N. 24, Ed. 247, Art. 1635, Dezembro, 2013.

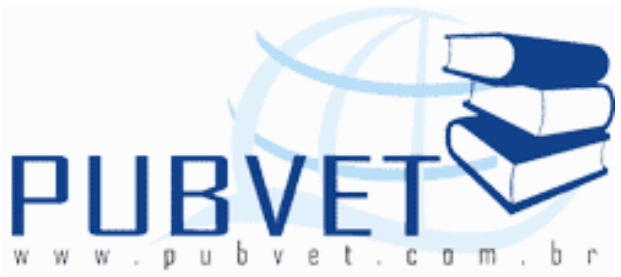

PUBVET, Publicações em Medicina Veterinária e Zootecnia.

\title{
Relação entre reprodução e produção de bovinos de leite
}

Kleber da Cunha Peixoto Junior ${ }^{1}$, Angelina Cecilia Firmiano², André Maciel Crespilho ${ }^{3}$

${ }^{1}$ Professor Doutor da UNISA, UNIP e UMESP

${ }^{2}$ Aluna de graduação da UMESP

${ }^{3}$ Professor Doutor da UNISA

\section{Resumo}

A fertilidade de vacas leiteiras tem diminuído ao longo das últimas cinco décadas e a produção de leite por vaca aumentado. Embora as dificuldades em alcançar índices reprodutivos satisfatórios em rebanhos de alta produção estejam associadas a uma série fatores como o aumento no tamanho do rebanho, mudanças nas condições de instalação, falhas na inseminação artificial, dificuldade de observação de cios, diminuíção ou supressão do comportamento de estro devido ao tipo de piso, inibição social, calor intenso, barulho e estresse pelo calor. Para a maioria dos pesquisadores, a principal causa atribuida a relação deleteria entre produção de leite e reprodução é o Balanço Energetico Negativo ocasionado pelo parto, início da lactação e aumento da demanda por energia e proteínas para atender a produção de leite. No período pós parto, ocorrem ainda alterações metabólicas que originam modificações na produção de membros da família IGF, além do atraso na recuperação da secreção de leptina. Falhas na detecção de cio que muitas 
PEIXOTO JUNIOR, K.C., FIRMIANO, A.C. e CRESPILHO, A.M. Relação entre reprodução e produção de bovinos de leite. PUBVET, Londrina, V. 7, N. 24, Ed. 247, Art. 1635, Dezembro, 2013.

vezes pode ser mais discreto e curto estão relacionadas aos problemas reprodutivos em função de altos indices de produção leiteira. Esta revisão de literatura tem por objetivo levantar os efeitos negativos da produção de leite sobre a eficiência reprodutiva de bovinos leiteiros de alta produção.

Palavras-chave: Produção de leite, Reprodução, Balanço Energetico Negativo, vacas.

\title{
The relationship between production and reproduction of dairy cattle
}

\begin{abstract}
The fertility of dairy cows has declined over the past five decades and milk production per cow increased. The difficulties to reach satisfactory reproductive performance in high producing herds is associated with a series factors such as the increase in herd size, changes in the conditions of installation, artificial insemination failure, difficulty watching ness reduction or suppression of estrus behavior because the type of floor, social inhibition, intense heat and noise stress, for most researchers negative energy balance is the major cause attributed to deleterious relationship between milk production and reproduction are caused by childbirth, early lactation and increase of demand for energy and protein to attend milk production. In the postpartum period, there are also metabolic changes which give rise to the production of IGF family members in addition to the delayed recovery of the secretion of leptin. Failure to detect estrus can often be more discreet and brief are related to reproductive problems because of high rates of milk production. This literature review aimed to investigate the negative effects of milk production on reproductive performance of high producing dairy cattle.
\end{abstract}

Keywords: Milk production, Reproduction, Negative Energy Balance, cows. 
PEIXOTO JUNIOR, K.C., FIRMIANO, A.C. e CRESPILHO, A.M. Relação entre reprodução e produção de bovinos de leite. PUBVET, Londrina, V. 7, N. 24, Ed. 247, Art. 1635, Dezembro, 2013.

\section{INTRODUÇÃO}

A bovinocultura de leite no Brasil vem apresentando significativo crescimento nos últimos anos. Segundo dados do IBGE em 2000 o Brasil produziu 19,8 milhões de litros, em 2009 chegou a 29,1 milhões de litros, em 2012 atingiu a marca de 32 milhões de litros produzidos em um total de 1,3 milhões de propriedades com rebanho produtivo de 23 milhões de animais.

A eficiência reprodutiva é o fator que, isoladamente, mais afeta a produtividade e a lucratividade de um rebanho. Entretanto, há muitos obstáculos para otimizá-la, pois ocorrem perdas reprodutivas desde a concepção (natural ou artificial) até o parto. Essas perdas irão afetar diretamente o sucesso da exploração causando forte impacto negativo sobre a rentabilidade da produção pecuária. Com a redução na produção de leite, haverá aumento do intervalo entre lactações, assim como prolongamento do período seco da vaca e da proporção de vacas secas no rebanho (EMPRESA BRASILEIRA DE PESQUISA AGROPECUÁRIA, 2010).

A fertilidade de vacas leiteiras tem diminuído ao longo das últimas cinco décadas e a produção de leite por vaca aumentou. Muitas hipóteses têm sido propostas para explicar estas questões, incluindo genética, fisiologia, nutrição e manejo, e esses fatores têm sido investigados nos animais em momentos críticos da vida produtiva, principalmente de vacas leiteiras (WALSH; WILLIAMS \& EVANS, 2011).

A presente revisão de literatura tem por objetivo levantar os efeitos negativos da produção de leite sobre a eficiência reprodutiva de bovinos leiteiros de alta produção. 
PEIXOTO JUNIOR, K.C., FIRMIANO, A.C. e CRESPILHO, A.M. Relação entre reprodução e produção de bovinos de leite. PUBVET, Londrina, V. 7, N. 24, Ed. 247, Art. 1635, Dezembro, 2013.

\section{REVISÃO DA LITERATURA}

O possível antagonismo entre alta produção de leite e desempenho reprodutivo é um dos principais assuntos discutidos entre produtores de leite e pesquisadores. Alguns se preocupam com a seleção genética para fertilidade, ou questionam se o manejo é capaz de satisfazer as necessidades das vacas em termos de alta produção e obtenção de prenhez no momento adequado. (LEBLANC, 2010).

A Eficiência reprodutiva é um fator importante que afeta a produção e eficiência econômica em rebanhos leiteiros (DISKIN , 2008). Em varios países durante muitos anos a criação de vacas leiteiras, teve como principal objetivo a seleção voltada para as características produtivas (SEWALEM \& KISTEMAKER, 2008). Há evidências crescentes e consistentes que sugerem que pelo menos uma parte do declínio no desempenho reprodutivo está relacionada a mudanças subjacentes na fisiologia reprodutiva causada por alta produção de leite e ou balanço energético negativo no início da lactação (DISKIN , 2008).

\subsection{Fatores que interferem na produção de leite e os índices de reprodução}

Nos últimos 30 anos, a seleção genética para produção de leite aumentou, resultando no aumento da taxa de lactação anual e no conseqüente aumento da incidência de problemas metabólicos e reprodutivos (KNOP E CERNESCU, 2009).

A dificuldade em alcançar índices reprodutivos satisfatórios em rebanhos leiteiros de alta produção está associado a uma serie fatores como o aumento no tamanho do rebanho, mudanças nas condições de instalação, falhas na inseminação artificial (RODRIGUEZ-MARTINEZ et al, 2008), dificuldade de observação de cios, diminuíção ou supressão do comportamento de estro 
PEIXOTO JUNIOR, K.C., FIRMIANO, A.C. e CRESPILHO, A.M. Relação entre reprodução e produção de bovinos de leite. PUBVET, Londrina, V. 7, N. 24, Ed. 247, Art. 1635, Dezembro, 2013.

devido ao tipo de piso, inibição social, calor intenso, barulho (LAN-DAETAHERNÁNDEZ et al. 2002), estresse pelo calor (FERREIRA, R. A.; 2005), doenças pós parto (BARROS, 2010), lesões de casco (DIAS E MARQUES, 2003), mastite (SANTOS et al. 2003) e manejo nutricional (CORRÊA et. al 2013).

Para Barros (2010), é freqüente o desenvolvimento simultâneo ou sucessivo de várias doenças associadas ao parto (DAP) tais como retenção placentária, metrite e endometrite, cetose e hipocalcemia, que agravam o quadro clínico e diminui o prognóstico de recuperação da saúde e da fertilidade das vacas.

Maiores influências do estresse calórico podem ser observadas em vacas das raças européias comparadas às de raças zebuínas. Altas temperaturas podem reduzir a taxa de fecundação e de manifestação de cio devido ao desvio do fluxo sanguíneo para a periferia do corpo do animal, levando a menores quantidades de sangue em regiões próximas ao útero, menor fornecimento de nutrientes e aumento da temperatura uterina (FERREIRA,R.A.; 2005). Para Costa-Silva et al. (2010), condições ambientais adversas tais como altas temperaturas e umidade elevada geram estresse calórico nos animais e como consequência subfertilidade e até mesmo infertilidade. $O$ estresse calórico produz ainda hormônios da família das prostaglandinas que em altos níveis na corrente sanguinea podem estar associados a abortos (FERREIRA, R. A.; 2005).

Conforme Ferreira, P. M. (2005), as manqueiras levam a perdas consideráveis na produção de leite situando-se em média, nos casos graves, em $20 \%$ da lactação, e perdas reprodutivas que podem reduzir a ocorrência e observação do cio e a taxa de concepção. Para Dias e Marques (2003), vacas com cascos lesionados apresentam índice de concepção mais baixo no primeiro serviço, maior incidência de cistos ovarianos, sinais discretos de cio e até mesmo anestro. 
PEIXOTO JUNIOR, K.C., FIRMIANO, A.C. e CRESPILHO, A.M. Relação entre reprodução e produção de bovinos de leite. PUBVET, Londrina, V. 7, N. 24, Ed. 247, Art. 1635, Dezembro, 2013.

Segundo Moore et al.,1991 um caso clínico de mastite antes do primeiro serviço aumenta o número de dias para a ocorrência do primeiro serviço e, consequentemente, dos dias em aberto comparando-se com animais sadios. $\mathrm{O}$ mesmo foi observado por Santos et al. (2003), ao constatarem que casos de mastite subclínica que antecedem o $1^{\circ}$ serviço geram atraso na manifestação do cio, aumentando o numero de dias para a ocorrencia da concepção.

De acordo com Corrêa et. al (2013), as necessidades nutricionais para equilíbrio homeostático, crescimento e produção leiteira têm prioridade sobre a reprodução, sendo necessário, desta forma, um aporte de nutrientes que atenda todas estas exigências antes que a puberdade, o retorno à ciclicidade pós-parto ou a manutenção da gestação ocorram.

A vaca leiteira é uma das espécies animais com maior exigência nutricional principalmente no período pós-parto quando, geralmente, se encontra em severo balanço energético negativo (BEN), com elevada competição por nutrientes entre produção de leite e outras funções fisiológicas, como a reprodução (PASA, 2011). Para este autor, os níveis nutricionais podem afetar o desenvolvimento e a função dos órgãos reprodutivos, além de acarretar alterações do funcionamento do sistema endócrino envolvido com a reprodução. Desta forma Lucy (2008) informa que é importante observar se as vacas leiteiras são inerentemente menos férteis ou se a infertilidade é simplesmente uma função do seu nível de produção e partição de nutrientes.

2.2 Influencia da produção de leite sobre parâmetros reprodutivos de bovinos leiteiros

Ao longo dos anos questiona-se, se a seleção de vacas leiteiras com enfoque no aumento da produção de leite tem em contrapartida afetado a fertilidade desses animais. Foram relatadas correlações genéticas e fenotípicas 
PEIXOTO JUNIOR, K.C., FIRMIANO, A.C. e CRESPILHO, A.M. Relação entre reprodução e produção de bovinos de leite. PUBVET, Londrina, V. 7, N. 24, Ed. 247, Art. 1635, Dezembro, 2013.

de baixa a moderada entre produção e reprodução, mas geralmente em direção desfavorável (PRYCE et al.; 2004). Segundo Marti \& Funk (1994), a herdabilidade estimada da fertilidade é menor que 5\%, comparada a 25 a 50\% para características produtivas.

Vários estudos têm demonstrado que a retomada da atividade ovariana tem sido retardada e que as taxas de concepção caíram significativamente de $55 \%$ para $40 \%$, por conseguinte, o intervalo de partos aumentou de cerca de 385 dias para 412 dias. A percentagem de vacas descartadas por causa de infertilidade aumentou de 5 para $8 \%$ por ano (KRUIF; LEROY; OPSOMER, 2008).

Washburn et al. (2002) analisaram 532 rebanhos de vacas Holandesas e 29 rebanhos de vacas Jersey em propriedades dos Estados Unidos no período de 1976 a 1999, tendo observado, ao longo dos anos, aumento da produção leiteira, do número de dias em aberto (passando de aproximadamente 120 dias em 1970 para 150 em 1999 nas vacas Jersey e 125 para 168 em vacas holandesas), do número de serviços por concepção (aumentando de 1,9 em 1976 para 2,9 em 1999 em vacas Jersey e 1,9 em 1976 para 3,0 em 1999 em vacas holandesas) e redução da taxa de concepção anual.

Riecka \& Candrák (2011), observaram entre os anos de 2003 e 2009 aumento na produção de leite e prolongamento no intervalo de partos durante este período, sugerindo que existem relações antagônicas entre produção de leite e fertilidade, resultados semelhantes foram encontrados por Lucy (2001), que, entre 1970 e 2000, avaliaram 143 rebanhos americanos e observaram que a produção de leite aumentou de $6500 \mathrm{~kg}$ para aproximadamente $9000 \mathrm{~kg}$ por lactação, que o período de serviço por concepção passou de quase 1,75 para 3,0 e o intervalo entre partos aumentou de aproximadamente 13,5 meses para 14,8 meses. 
PEIXOTO JUNIOR, K.C., FIRMIANO, A.C. e CRESPILHO, A.M. Relação entre reprodução e produção de bovinos de leite. PUBVET, Londrina, V. 7, N. 24, Ed. 247, Art. 1635, Dezembro, 2013.

Peixoto Jr et al. em estudo não publicado, avaliou o efeito da produção de leite sobre parâmetros reprodutivos de 1255 vacas leiteiras durante 11 meses e concluíram que elevada produção de leite no mês anterior à cobertura diminui a eficiência reprodutiva de vacas, já que esta apresentou efeito linear significativo sobre o período de serviço, número de coberturas por prenhez, intervalo entre o parto e a primeira inseminação e taxa de prenhez.

A principal causa atribuída à relação deletéria entre produção de leite e reprodução é o Balanço Energético Negativo (BEN). Este BEN ocorre devido à situação de estresse ocasionado pelo parto, início da lactação e aumento da demanda por energia e proteínas para atender a produção de leite associados a um consumo de matéria seca reduzido, geralmente insuficiente para atender as exigências de manutenção e produção (WALSH; WILLIAMS; EVANS, 2011).

Em vacas bem alimentadas, o saldos negativo de energia começa a melhorar após quatro semanas de lactação. Esta recuperação do balanço energético a partir de seu estado mais negativo pode ser um indício para o início da atividade ovariana. Isto sugere que o BEN pode comprometer a fertilidade, atrasando o primeiro cio e limitando o número de ciclos estrais pósparto (KNOP; CERNESCU, 2009).

Fenwick et al. (2008), analisaram o início da lactação de vacas de alta produção e observaram baixa concentração de IGF-I e maiores níveis de IGF-II circulante após o parto e sugeriram que o BEN pós parto pode influenciar a disponibilidade de IGF no oviduto indiretamente através de alterações na expressão de IGF-proteína de ligação específico e que o aumento de IGF-II pode prejudicar o desenvolvimento do embrião, contribuindo para as altas taxas de mortalidade embrionária em vacas leiteiras. Estes autores relatam que, no período pós parto, ocorrem alterações metabólicas que originam modificações importantes na produção de membros da família IGF, concluindo que concentrações reduzidas de IGF-I estão associados com baixa fertilidade. 
PEIXOTO JUNIOR, K.C., FIRMIANO, A.C. e CRESPILHO, A.M. Relação entre reprodução e produção de bovinos de leite. PUBVET, Londrina, V. 7, N. 24, Ed. 247, Art. 1635, Dezembro, 2013.

Para Knop e Cernescu (2009), o balanço energético negativo pode atrasar o primeiro cio limitando o número de ciclos estrais no pós-parto. Devido ao BEN, as concentrações de insulina permanecem baixas, o que impede um aumento nos receptores do hormônio de crescimento no fígado e a secreção de IGF-I fazendo com que o eixo somatotrófico seja alterado. Além disso, um atraso na recuperação da secreção de leptina aumenta o tempo para que ocorra a primeira ovulação (KADOKAWA; MARTIN, 2006), pois é muito provável que a leptina acompanhe IGF-I no retorno da ovulação sendo secretada principalmente pelo tecido adiposo, atuando como sinalizador metabólico no sistema nervoso central controlando a libertação "pulsátil" de LH (KNOP; CERNESCU, 2009).

A falha na detecção de cio que muitas vezes pode ser mais discreto e curto está relacionado aos problemas reprodutivos em função de altos indices de produção leiteira. Um estudo realizado por Lopez et al., (2004) procurou mostrar a influência da maior produtividade leiteira sob a duração do cio. Ao fim do experimento a produção de leite foi correlacionada com a duração do cio, onde maiores lactações apresentaram concentrações mais baixas de estradiol. Foi concluído pelo estudo que a duração do estro é reduzida por um aumento do nivel de produção de leite, sugerindo que as concentrações de estradiol no estro e a duração e intensidade do estro estão inversamente influenciadas na produção de leite fornecendo assim uma explicação parcial para as baixas taxas de detecção de cio.

Kruif; Leroy; Opsomer (2008), a "síndrome da infertilidade" é um problema multifatorial, causado inicialmente pelo o período de balanço energético negativo e nutrição que afetam a sinalização endócrina, o crescimento folicular e, provavelmente, também a qualidade ovócito e do embrião. Para estes autores, as soluções para este problema complexo é difícil já que envolvem melhorias de uma série de fatores críticos como instalações, deteç̧ão de cio, manejo nutricional pré e pós parto e períodos de lactação, 
PEIXOTO JUNIOR, K.C., FIRMIANO, A.C. e CRESPILHO, A.M. Relação entre reprodução e produção de bovinos de leite. PUBVET, Londrina, V. 7, N. 24, Ed. 247, Art. 1635, Dezembro, 2013.

manejo da inseminação artificial, higiene e cuidado em torno do parto, além de utilização de semen de boa qualidade.

No entanto, para alguns autores, a produção de leite em si, não prejudica a eficiência reprodutiva do rebanho, cabendo ao manejo dos animais a maior importância. Leblank (2010), observou taxas de prenhez significantemente mais elevada em rebanhos de maior produção, observandose pequenos efeitos do nível de produção sobre o intervalo entre partos. Para estes autores, um bom manejo voltado para a alta produção é compatível com o bom desempenho reprodutivo. Lopez-Gatuis et al. (2006) avaliaram 2.756 prenhezes em dois rebanhos de alta produção na Espanha e observaram que vacas com produção média de $49.5 \mathrm{~kg} /$ dia de leite estavam prenhes aos 90 dias de lactação, em contrapartida vacas com 43,2 kg/dia emprenharam mais tarde. Steverson (1999) sugeriu, ao avaliar 1,2 milhões de vacas da raça Holandesa em 9684 rebanhos e 50.000 vacas Jersey de 546 rebanhos, que há um melhor aproveitamento reprodutivo em rebanhos com maior media de produção de leite e que isto pode ser um reflexo de melhor alimentação, de vacas mais saudáveis e melhor gestão no manejo reprodutivo.

\section{CONCLUSÃO}

Conclui-se que elevadas produções de leite interferem na taxa reprodutiva de rebanhos leiteiros, já que agravam o BEN que interfere no decréscimo dos índices reprodutivos. 
PEIXOTO JUNIOR, K.C., FIRMIANO, A.C. e CRESPILHO, A.M. Relação entre reprodução e produção de bovinos de leite. PUBVET, Londrina, V. 7, N. 24, Ed. 247, Art. 1635, Dezembro, 2013.

\section{REFERÊNCIAS}

BARROS, L. N. F. Relação entre doença associada ao parto (DAP) e parâmetros biológicos, metabólicos e de fertilidade em vacas de elevada produção leiteira. Dissertação de Mestrado. Universidade Técnica de Lisboa, Faculdade de Medicina Veterinária, Lisboa.2010. Disponivel em:

<https://www.repository.utl.pt/bitstream/10400.5/2210/1/Rela\%c3\%a7\%c3\%a30\%20entre\% 20Doen\%c3\%a7a\%20Associada\%20ao\%20Parto\%20DAP\%20e\%20par\%c3\%a2metros\%20bio 1\%c3\%b3gicos\%20metab\%c3\%b3licos\%20e\%20de\%20fertilidade\%20em\%20vacas\%20de $\% 2$ 0elevada\%20produ\%c3\%a7\%c3\%a3o\%20leiteira.pdf> Acesso em: 10 de março de 2013.

CORRÊA, M.N., et al. Transtornos metabólicos no Gado Leiteiro: Interação entre Sanidade, Nutrição e Reprodução. Disponível em: < http://www.petsa.com.br/uploads/RESUMOS_OK/073.pdf>. Acesso em: 02 de março de 2013.

COSTA-E-SILVA, E. V. et al. Estratégias para avaliar bem-estar animal em animais em reprodução. 2010.

DIAS, R.O.S; MARQUES JR, P. Atlas de Cascos em Bovinos, $2^{a}$ ed. São Paulo: Lemos Editora, 2003. 67p.

DISKIN, Michael G. Reproductive management of dairy cows: a review (part I). Irish Veterinary Journal, v. 61, n. 5, p. 326, 2008.

EMBRAPA. Eiciência reprodutiva das vacas leiteiras. Circular Técnica, 64. 2010 Disponível em: < http://ainfo.cnptia.embrapa.br/digital/bitstream/item/

29218/1/Circular64-2.pdf>. Acesso em: 31 fev. 2013.

FERREIRA, P.M., Carvalho, A.U., Facury Filho, E.J.,Ferreira, M.G. and Ferreira, R.G. 2005. Afecções do sistema locomotor dos bovinos. Proceedings of $2^{\circ}$ Simpósio Mineiro de Buiatria. Disponivel em:http://www.ivis.org/proceedings/

abmg/ 2005/pdf04.pdf (10-2-2013).> Acesso em: 15 de abril de 2013.

FERREIRA, R. A. Maior Produção com Melhor Ambiente para aves, suínos e bovinos. 1.ed. Viçosa: Aprenda Fácil, 2005. 371p.

FENWICK, M. A. et al. Negative energy balance in dairy cows is associated with specific changes in IGF-binding protein expression in the oviduct. Reproduction, v. 135, n. 1, p. 6375, 2008.

IBGE. INSTITUTO BRASILEIRO DE GEOGRAFIA E ESTATÍSTICA / Pesquisa da Pecuária Municipal e Censo Agropecuário. SIDRA. Disponível em: <www.sidra.ibge.gov.br> Acesso: Fevereiro 2013.

KADOKAWA, H., MARTIN, B. G., A new perspective on Management of reproduction in dairy cows: the need for detailed metabolic information, an improved selection index and extended lactation, J. Reprod. Dez., 2006, 52, 1, 161-168.

KNOP, R., CERNESCU H. Effects of negative energy balance on reproduction in dairy cows. Lucrări Stiinłifice Medicină Veterinară, v. 42, n. 2, p. 198-205, 2009. 
KRUIF DE A; LEROY, J; OPSOMER, G. Reproductive performance in high producing dairy cows:practical implications.2008. Disponível em:

<https://biblio.ugent. be/input/download?func=downloadFile\&recordOId =822574\&fileOId $=831$ 283 > Acesso em: 14 de abril de 2013

LANDAETA-HERNANDEZ, A. J.; YELICH, J.; LEMASTER, J. W. Environmental, genetic and social factors affecting the expression of estrus in beef cows. Theriogenology, v. 57, p. 1357-1370, 2002.

LEBLANC, S. Assessing the association of the level of milk production with reproductive performance in dairy cattle. Journal of Reproduction and Development, v. 56, n. S, p. 17, 2010.

LOPEZ, H.; SATTER, L. D.; WILTBANK, M. C. Relationship between level of milk production and estrous behavior of lactating dairy cows. Animal reproduction science, v. 81, n. 3, p. 209223, 2004.

LÓPEZ-GATIUS et al. Screening for high fertility in high-producing dairy cows.

Theriogenology, v. 65, n. 8, p. 1678-1689, 2006.

LUCY, M.C. Functional differences in the growth hormone and insulin-like growth factor axis in cattle and pigs: implications for post-partum nutrition and reproduction. Reprod. Domest. Anim.43:31-39; 2008.

LUCY, M.C. Reproductive loss in high-producing dairy cattle: Where will it end? J. Dairy Sci.; 84:1277-1293, 2001.

MARTI, C.F; FUNK, D.A. Relationship between production and days open at different levels of herd production. J.Dairy Sci.; 77:1682-1690; 1994

MOORE, D.A; CULLOR, R.H; SISCHO, W.M. Preliminary field evidence for the association of clinical mastitis with altered us intervals in dairy cattle. Theriogenology, v.36, p.257-265, 1991.

PASA, C. Relação reprodução animal e os minerais. Biodiversidade, v. 9, n. 1, 2011.

PRYCE, J.E et al. Fertility in the high producing dairy cow. Livest. Prod. Sci.2004; 86:125135.

RIECKA, Z; CANDRÁK, J. Analysis of Relationship between production and Reproduction Traits of Holstein Cattle Population in the Slovak Republic. Scientific papers Faculty of Animal Sciences and Biotechnologies, Timisoara, v. 44, n. 1, p. 332-336, 2011.

RODRIGUEZ-MARTINEZ, H., et al. Reproductive Performance in High-producing Dairy Cows: Can We Sustain it Under Current Practice?International Veterinary Information Service, Ithaca, NY. (Eds.), 2008

SANTOS, J.E.P., CERRI, R.L.A; KIRK, J.H. Effect of clinical mastitis incidence on lactation and reproductive performance of Holstein dairy cows. Animal Reproduction Science, v.58, p.312-327, 2003. 
SEWALEM, A; KISTEMAKER, G. Including production in female fertility evaluations. Interbull Bulletin, n. 38, p. 44, 2008.

STEVENSON J.S. Can you have good reproduction and high milk yield? Hoard's Dairyman 144: 536,1999 .

WALSH, S. W.; WILLIAMS, E. J.; EVANS, A. C. O. A review of the causes of poor fertility in high milk producing dairy cows. Animal reproduction science, v. 123, n. 3, p. 127-138, 2011.

WASHBURN, S. P. et al. Trends in reproductive performance in southeastern Holstein and Jersey DHI herds. Journal of dairy science, v. 85, n. 1, p. 244-251, 2002. 